\title{
PGRN alleviates cerebral amyloid- $\beta$ burden and cognitive impairments via mediating neuroinflammation
}

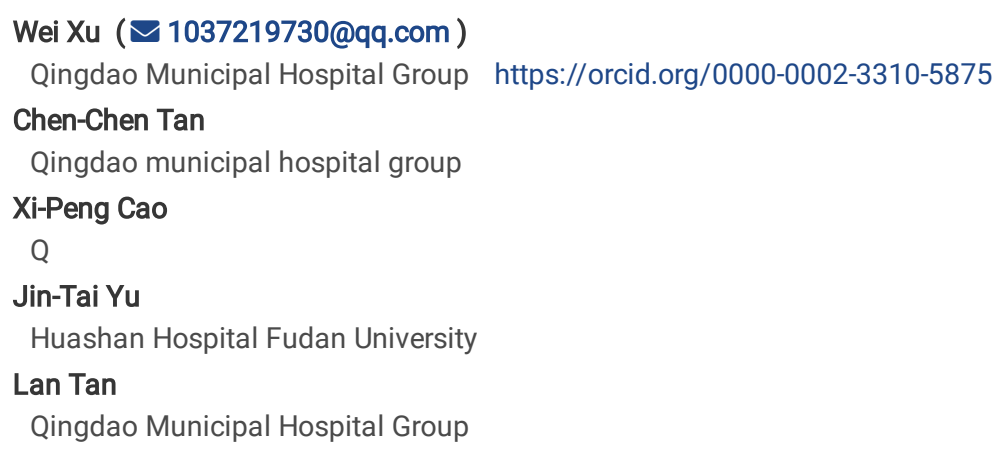

\section{Research}

Keywords: Progranulin, Neuroinflammation, Alzheimer, Amyloid, Tau, Cognition

Posted Date: July 22nd, 2020

DOl: https://doi.org/10.21203/rs.3.rs-44700/v1

License: (c) (i) This work is licensed under a Creative Commons Attribution 4.0 International License. Read Full License 


\section{Abstract \\ Background}

Both progranulin (PGRN) and neuroinflammatory activities increased over the course of Alzheimer's disease (AD). In this study, we set out to determine if cerebrospinal fluid (CSF) PGRN could be a marker of neuroinflammation, and if so, how it contributed to AD pathogenesis and cognitive impairments.

\section{Methods}

A total of 965 participants from the Alzheimer's Disease Neuroimaging Initiative (ADNI) were grouped within the framework of A-T-N biomarker profile and clinical stage. Causal mediation analyses with 10,000 bootstrapped iterations were conducted to explore the mediation effects of neuroinflammatory markers on the associations of PGRN with amyloid burden indicated by CSF $\beta$-amyloid (A $\beta$ ) levels. The longitudinal influences of PGRN on cognition were tested.

\section{Results}

CSF PGRN and multiple neuroinflammatory markers (sTNFR1, sTNFR2, TGF- $\beta 1$, VCAM1, and ICAM1) were increased with tau-related neurodegeneration. PGRN was positively linked with neuroinflammatory markers associated with tau pathologies (TN+). In TN + population, PGRN was associated with higher CSF A 42 via mediating neuroinflammatory markers and could predict slower cognitive decline. The abovementioned associations became nonsignificant in TN-profile.

\section{Conclusions}

These preliminary findings supported that PGRN could protect against $A \beta$ pathology and cognitive impairments via modulating neuroinflammation that occurs with neuronal injuries.

\section{Background}

Progranulin (PGRN) is a secreted glycoprotein expressed in the central nervous system (CNS). Its deficiency has been associated with lysosomal function [1, 2], autophagy [3], neuroinflammation [4, 5], and neurodegenerative diseases [1, 2], such as Alzheimer's disease (AD) [6, 7]. Neuroinflammation plays a critical role in AD [8]. We previously reported that levels of multiple inflammatory markers (e.g., soluble tumor necrosis factor receptor [sTNFRs], intercellular cell adhesion molecule-1 [ICAM1], and vascular cell adhesion molecule-1 [VCAM1]) in the cerebrospinal fluid (CSF) were significantly elevated in AD patients compared to healthy controls [9]. Interestingly, CSF PGRN was also found to be increased over the course of AD [10]. However, little is known about the associations of PGRN with these neuroinflammation markers. Additionally, the impacts of PGRN on AD pathologies, especially $\beta$-amyloid $(A \beta)$, was disputable in in-vivo or in-vitro studies. $A \beta$ plaques with PGRN were identified in low-plaque individuals, suggesting PGRN was involved in early plaque formation. Some reported that PGRN showed positive [11] or no [4] correlations with A 3 , while others found PGRN overexpression protected against $A \beta$ deposition and toxicity [12]. The cause of these controversial findings warrants further investigation in humans. Furthermore, little is known about whether neuroinflammation could mediate the associations between PGRN and AD pathologies.

In the present study, we aimed to i) examine whether PGRN could reflect inflammatory activity in CNS, ii) test the roles of neuroinflammation in mediating the influences of PGRN on amyloid pathologies, and iii) test the longitudinal influences of PGRN on cognitive functions. All analyses were conducted within the framework of A-T-N biomarker profile using the Alzheimer's Disease Neuroimaging Initiative (ADNI).

\section{Methods}

\subsection{Study participants}

ADNI is designed to develop clinical, imaging, genetic, and biochemical biomarkers for the early detection and tracking of AD. The participants are volunteers aged 55-90 years with normal or impaired cognition. Further information can be found at http://www.adni-info.org/ and in previous reports [13-15]. At baseline, each participant underwent an in-person interview of general health and functional ability, followed by a standardized assessment including a battery of neuropsychological tests. Follow-up data were collected during evaluations at sequential intervals of approximately 12 months. ADNI was approved by institutional review boards of all participating institutions, and written informed consent was obtained from all participants or their guardians.

In the present study, a total of 965 participants who had baseline measurements of CSF PGRN and AD core biomarkers, as well as longitudinal measurements of cognitive functions were included. Among these individuals, 228 had measurements of CSF neuroinflammatory markers.

\subsection{Classification methods}


The classification methods were in line with those proposed by Marc Suárez-Calvet et al. [10, 16] according to 2018 NIA-AA "research framework" for AD diagnosis [17]. In brief, participants were categorized into specific groups based on both biomarker profile (as described by the A/T/N scheme [18]) and clinical stage (as defined by the clinical dementia rating [CDR] score). The A/T/N scheme includes 3 biomarker groups: " $\mathrm{A}$ " aggregated amyloid pathology (as indicated by CSF $A \beta_{1-42}$ ), " $T$ " aggregated tau (as indicated by CSF p-tau ${ }_{181}$ ), and " $\mathrm{N}$ " neurodegeneration or neuronal injury (as indicated by CSF t-tau). "At" participants refer to those with CSF $A \beta_{1-42}<976.6 \mathrm{pg} / \mathrm{ml}$; "T+" those with CSF p-tau $181>21.8 \mathrm{pg} / \mathrm{ml}$; and "N+" those with CSF t-tau >

$245 \mathrm{pg} / \mathrm{ml}$. The CSF biomarker statuses established by these cutoffs were proven to be highly concordant with PET classification in ADNI [19]. Given that $\mathrm{T}$ and $\mathrm{N}$ groups were highly correlated, we merged them together to facilitate the analyses, producing a TN group: "TN+" indicates $\mathrm{T}+\mathrm{or} \mathrm{N}+$ and "TN-" indicates $\mathrm{T}$ - and $\mathrm{N}$ -

\subsection{CSF measurements of PGRN, neuroinflammatory markers, and AD core biomarkers}

CSF procedural protocols in ADNI have been described previously [20]. CSF PGRN was measured by a previously reported sandwich immunoassay using the Meso Scale Discovery platform [21]. All CSF samples were distributed randomly across plates and measured in duplicate. All the antibodies and plates were from a single lot in order to exclude variability between batches. The mean intraplate coefficient of variation (CV) was $2.2 \%$; all duplicate measures had a CV $<15 \%$. PGRN levels were corrected by inter-batch variation and corrected values were used for analyses (for the method see Appendix 1).

CSF AD core biomarkers, including $A \beta_{1-42}, p$-tau 181 , and t-tau were analyzed by the electrochemiluminescence immunoassays (ECLIA) Elecsys on a fully automated Elecsys cobas e 601 instrument and a single lot of reagents for each of the three measured biomarkers (provided in UPENNBIOMK9.csv file). These measurements are for explorative research use only. A total of 8 types of CSF neuroinflammatory markers, including four anti-inflammatory markers (sTNFR1, sTNFR2, TGF- $\beta 1$, and IL-10) and four pro-inflammatory markers (ICAM1, VCAM1, IL-6, and IL-7) were measured, using commercially available multiplex immunoassays (Millipore Sigma, Burlington, MA). All samples were run in duplicate along with six standards on each plate. Samples were normalized across plates using CSF standard values. Precision of each analyte was calculated using inter-plate CV $<15 \%$.

\subsection{Cognitive measures}

Global cognitive function was reflected by the total scores of Alzheimer's Disease Assessment Scale (ADAS). Composite scores for executive and memory functions were constructed and validated by referring to the neuropsychological batteries [22, 23]. Specifically, the indicators of executive functions include Category Fluency, WAIS-R Digit Symbol, Trails A \& B, Digit Span Backwards, and clock drawing. The indicators of memory function include relevant items of the Rey Auditory Verbal Learning Test (RAVLT), ADAS, Logical Memory, and Mini-Mental State Examination (MMSE). The CDR score was used to represent the clinical stage: " 0 " represents normal cognition, " 0.5 " represents very mild dementia, and " 1 " represents mild dementia.

\subsection{Statistical analysis}

Before the analyses, values of CSF markers as dependent variables were $\log _{10}$-transformed to achieve or approximate normal distributions as assessed by Kolmogorov-Smirnov test. All analyses were adjusted for age, gender, educational level, and CDR, except where specifically noted.

First, one-way analyses of covariance (ANCOVAs) were separately performed to examine the associations of CSF PGRN and neuroinflammatory markers with A/TN status, within the framework combining A/TN classification and clinical stage. Four comparisons were separately conducted for each CDR group, including $A-/ T N+v s$. A-/TN-, A+/TN + vs. A+/TN- (the former two comparisons were to test the associations of tau-related neurodegeneration with biomarkers), $A+/ T N+v s$. A-/TN+, and A+/TN- vs. A-/TN- (the latter two were for the associations with amyloid pathology). Next, multiple linear regressions were conducted to explore the associations of CSF PGRN (an independent variable) with neuroinflammatory markers (dependent variables) within the A/TN framework.

Further, we tested whether PGRN could modulate CSF $A \beta_{1-42}$ via mediating specific neuroinflammatory markers. To achieve this, causal mediation analyses were conducted using linear regression models fitted based on the methods proposed by Baron and Kenny [24]. The direct effects, indirect effects, and the mediating proportion were estimated by Sobel's test [25] with the significance determined using 10,000 bootstrapped iterations.

Finally, linear mixed effects (LME) models were used to estimate the longitudinal influences of CSF PGRN on cognitive functions. To facilitate the depiction, CSF PGRN was categorized into three groups (low, moderate, and high) using cutoffs of 1,396 pg/ml and 1,684 pg/ml according to the tertiles of the concentration. The LME models had random intercepts and slopes for time and an unstructured covariance matrix for the random effects, and included the interaction between time (continuous) and the dependent variable (PGRN) as a predictor.

Sensitivity analyses were conducted as follows. a) We repeated the analyses after excluding outlier values of CSF markers, defined as values situated outside the 3 standard deviations from the mean; b) rs5848 genotype of GRN gene, which was associated with PGRN levels, was added as a covariate in analyses with CSF PGRN as the dependent variable. The results barely changed after these analyses. R version 3.5.1 (major packages include "Im", "ggplot2", "mediate", and "nlme") and GraphPad Prism 7.00 software were used for statistical analyses and figure preparation. All tests were two-tailed, with a significance level of $a=0.05$.

\section{Results}




\subsection{Increase of PGRN was associated with tau-related neurodegeneration, but not with $\beta$-amyloid.}

We separately studied the associations of CSF PGRN with A 3 deposition and tau-related neurodegeneration within the framework combining A/TN classification and clinical stage in 965 participants from the ADNI (Table 1). The combination of A/TN classification and clinical stage divided subjects into 12 different groups that are summarized in Table e-1. 
Table 1

Demographic and clinical characteristics of the participants

\begin{tabular}{|c|c|c|c|c|c|c|c|c|c|c|c|c|}
\hline \multirow[t]{2}{*}{$N=965$} & \multicolumn{4}{|l|}{$\mathrm{CDR}=0$} & \multicolumn{4}{|c|}{$\mathrm{CDR}=0.5$} & \multicolumn{4}{|l|}{$\mathrm{CDR}=1$} \\
\hline & $\begin{array}{l}\text { A-/TN- } \\
(n= \\
121)\end{array}$ & $\begin{array}{l}A+T N- \\
(n=52)\end{array}$ & $\begin{array}{l}\text { A+/TN+ } \\
(n=45)\end{array}$ & $\begin{array}{l}\text { A-/TN+ } \\
(n=73)\end{array}$ & $\begin{array}{l}\text { A-/TN- } \\
(n= \\
118)\end{array}$ & $\begin{array}{l}A+T N- \\
(n=89)\end{array}$ & $\begin{array}{l}\text { A+/TN+ } \\
(n= \\
270)\end{array}$ & $\begin{array}{l}\text { A-/TN+ } \\
(n=97)\end{array}$ & $\begin{array}{l}A-/ T N- \\
(n=2)\end{array}$ & $\begin{array}{l}\text { A-TN- } \\
(n= \\
12)\end{array}$ & $\begin{array}{l}A+/ T N+ \\
(n=78)\end{array}$ & $\begin{array}{l}A-/ T N+ \\
(n=8)\end{array}$ \\
\hline Age & $\begin{array}{l}72.5 \\
(5.4)\end{array}$ & $\begin{array}{l}73.2 \\
(6.1)\end{array}$ & $\begin{array}{l}76.5 \\
(5.5)\end{array}$ & $\begin{array}{l}74.5 \\
(6.6)\end{array}$ & $\begin{array}{l}69.7 \\
(7.6)\end{array}$ & $\begin{array}{l}72.5 \\
(7.7)\end{array}$ & $\begin{array}{l}73.3 \\
(7.1)\end{array}$ & $\begin{array}{l}73.2 \\
(8.1)\end{array}$ & $\begin{array}{l}89.2 \\
(1.6)\end{array}$ & $\begin{array}{l}77.9 \\
(3.7)\end{array}$ & $\begin{array}{l}73.8 \\
(9.3)\end{array}$ & $\begin{array}{l}79.9 \\
(7.4)\end{array}$ \\
\hline $\begin{array}{l}\text { Female, } \mathrm{n} \\
(\%)\end{array}$ & $\begin{array}{l}58 \\
(47.9)\end{array}$ & $\begin{array}{l}24 \\
(46.2)\end{array}$ & $\begin{array}{l}23 \\
(51.1)\end{array}$ & $\begin{array}{l}41 \\
(56.2)\end{array}$ & $\begin{array}{l}53 \\
(44.9)\end{array}$ & $\begin{array}{l}20 \\
(22.5)\end{array}$ & $\begin{array}{l}117 \\
(43.3)\end{array}$ & $\begin{array}{l}43 \\
(44.3)\end{array}$ & $0(0)$ & $\begin{array}{l}4 \\
(33.3)\end{array}$ & $\begin{array}{l}40 \\
(51.3)\end{array}$ & $2(25)$ \\
\hline $\begin{array}{l}\text { APOE4 } \\
\text { carriers, n } \\
(\%)\end{array}$ & $\begin{array}{l}16 \\
(13.2)\end{array}$ & $\begin{array}{l}22 \\
(42.3)\end{array}$ & $\begin{array}{l}26 \\
(57.8)\end{array}$ & $\begin{array}{l}16 \\
(21.9)\end{array}$ & $\begin{array}{l}25 \\
(21.2)\end{array}$ & $\begin{array}{l}43 \\
(48.3)\end{array}$ & $\begin{array}{l}206 \\
(76.3)\end{array}$ & 32 (33) & $0(0)$ & $6(50)$ & $\begin{array}{l}58 \\
(74.4)\end{array}$ & $1(12.5)$ \\
\hline $\begin{array}{l}\text { Education, } \\
\text { y }\end{array}$ & $\begin{array}{l}16.3 \\
(2.8)\end{array}$ & $\begin{array}{l}16.4 \\
(2.8)\end{array}$ & $\begin{array}{l}16.6 \\
(2.5)\end{array}$ & $\begin{array}{l}16.4 \\
(2.5)\end{array}$ & $\begin{array}{l}16.1 \\
(2.7)\end{array}$ & $\begin{array}{l}16.3 \\
(2.8)\end{array}$ & $\begin{array}{l}15.8 \\
(2.9)\end{array}$ & $\begin{array}{l}15.9 \\
(2.7)\end{array}$ & $17(0)$ & $\begin{array}{l}16.1 \\
(2.8)\end{array}$ & $\begin{array}{l}15.1 \\
(2.8)\end{array}$ & $\begin{array}{l}14.9 \\
(2.0)\end{array}$ \\
\hline \multicolumn{13}{|c|}{ CSF measurements, mean (SD), pg/ml } \\
\hline PGRN & $\begin{array}{l}1500 \\
(291)\end{array}$ & $\begin{array}{l}1424 \\
(358)\end{array}$ & $\begin{array}{l}1572 \\
(307)\end{array}$ & $\begin{array}{l}1756 \\
(357)\end{array}$ & $\begin{array}{l}1543 \\
(358)\end{array}$ & $\begin{array}{l}1464 \\
(292)\end{array}$ & $\begin{array}{l}1531 \\
(324)\end{array}$ & $\begin{array}{l}1700 \\
(328)\end{array}$ & $\begin{array}{l}1885 \\
(490)\end{array}$ & $\begin{array}{l}1726 \\
(403)\end{array}$ & $\begin{array}{l}1634 \\
(339)\end{array}$ & $\begin{array}{l}1974 \\
(475)\end{array}$ \\
\hline$A \beta 42$ & $\begin{array}{l}1447 \\
(251)\end{array}$ & $\begin{array}{l}748 \\
(144)\end{array}$ & $\begin{array}{l}709 \\
(168)\end{array}$ & $\begin{array}{l}1760 \\
(676)\end{array}$ & $\begin{array}{l}1396 \\
(263)\end{array}$ & $\begin{array}{l}479 \\
(207)\end{array}$ & $\begin{array}{l}610 \\
(151)\end{array}$ & $\begin{array}{l}1637 \\
(464)\end{array}$ & n.a. & $\begin{array}{l}492 \\
(116)\end{array}$ & $\begin{array}{l}513 \\
(125)\end{array}$ & $\begin{array}{l}1147 \\
(0)\end{array}$ \\
\hline$P-\operatorname{tau}_{181 p}$ & $\begin{array}{l}17.1 \\
(2.6)\end{array}$ & $\begin{array}{l}16.0 \\
(3.2)\end{array}$ & $\begin{array}{l}33.9 \\
(9.7)\end{array}$ & $\begin{array}{l}26.5 \\
(3.9)\end{array}$ & $\begin{array}{l}16.2 \\
(2.7)\end{array}$ & $\begin{array}{l}16.6 \\
(4.2)\end{array}$ & $\begin{array}{l}37.9 \\
(12.0)\end{array}$ & $\begin{array}{l}29.8 \\
(12.0)\end{array}$ & n.a. & $\begin{array}{l}18.1 \\
(2.8)\end{array}$ & $\begin{array}{l}36.7 \\
(14.9)\end{array}$ & $70.5(0)$ \\
\hline T-tau & $\begin{array}{l}194 \\
(28)\end{array}$ & $\begin{array}{l}172 \\
(31)\end{array}$ & $\begin{array}{l}334 \\
(86)\end{array}$ & $\begin{array}{l}300 \\
(47)\end{array}$ & $\begin{array}{l}187 \\
(30)\end{array}$ & $\begin{array}{l}174 \\
(34)\end{array}$ & $\begin{array}{l}365 \\
(101)\end{array}$ & $\begin{array}{l}324 \\
(96)\end{array}$ & n.a. & $\begin{array}{l}200 \\
(26)\end{array}$ & $\begin{array}{l}362 \\
(110)\end{array}$ & $718(0)$ \\
\hline \multirow[t]{2}{*}{$N=228$} & $\mathrm{CDR}=0$ & $\begin{array}{l}\mathrm{CDR}= \\
0.5\end{array}$ & $\mathrm{CDR}=1$ & $\mathrm{CDR}=0$ & $\begin{array}{l}\mathrm{CDR}= \\
0.5\end{array}$ & $\mathrm{CDR}=1$ & $\mathrm{CDR}=0$ & $\begin{array}{l}\mathrm{CDR}= \\
0.5\end{array}$ & $\begin{array}{l}\mathrm{CDR}= \\
1\end{array}$ & $\begin{array}{l}\mathrm{CDR}= \\
0\end{array}$ & $\begin{array}{l}\mathrm{CDR}= \\
0.5\end{array}$ & $\mathrm{CDR}=1$ \\
\hline & $\begin{array}{l}\text { A-/TN- } \\
(n=34)\end{array}$ & $\begin{array}{l}\text { A-/TN+ } \\
(n=9)\end{array}$ & $\begin{array}{l}A+/ T N+ \\
(n=14)\end{array}$ & $\begin{array}{l}\text { A-/TN+ } \\
(n=12)\end{array}$ & $\begin{array}{l}\text { A-/TN- } \\
(n=14)\end{array}$ & $\begin{array}{l}A-/ T N+ \\
(n=13)\end{array}$ & $\begin{array}{l}A+/ T N+ \\
(n=83)\end{array}$ & $\begin{array}{l}A-/ T N+ \\
(n=20)\end{array}$ & $\begin{array}{l}\text { A-/TN- } \\
(n=0)\end{array}$ & $\begin{array}{l}\text { A-/TN+ } \\
(n=5)\end{array}$ & $\begin{array}{l}A+/ T N+ \\
(n=23)\end{array}$ & $\begin{array}{l}\text { A-/TN+ } \\
(n=1)\end{array}$ \\
\hline Age & $\begin{array}{l}75.4 \\
(4.5)\end{array}$ & $\begin{array}{l}72.8 \\
(6.0)\end{array}$ & $\begin{array}{l}78.8 \\
(5.2)\end{array}$ & $\begin{array}{l}76.0 \\
(9.3)\end{array}$ & $\begin{array}{l}73.2 \\
(9.3)\end{array}$ & $\begin{array}{l}73.0 \\
(4.6)\end{array}$ & $\begin{array}{l}74.3 \\
(7.2)\end{array}$ & $\begin{array}{l}76.3 \\
(8.0)\end{array}$ & n.a. & $\begin{array}{l}79.7 \\
(4.8)\end{array}$ & $\begin{array}{l}74.2 \\
(4.8)\end{array}$ & $84.7(0)$ \\
\hline $\begin{array}{l}\text { Female, } n \\
(\%)\end{array}$ & $\begin{array}{l}15 \\
(44.1)\end{array}$ & $2(22.2)$ & $6(42.9)$ & $6(50)$ & $5(35.7)$ & $3(23.1)$ & $\begin{array}{l}40 \\
(48.2)\end{array}$ & 7 (35) & n.a. & $2(40)$ & $\begin{array}{l}11 \\
(47.8)\end{array}$ & 1 (100) \\
\hline $\begin{array}{l}\text { APOE4 } \\
\text { carriers, } \mathrm{n} \\
(\%)\end{array}$ & $5(14.7)$ & $4(44.4)$ & $8(57.1)$ & 1 (8.3) & $3(21.4)$ & 8 (61.5) & $58(70)$ & $5(25)$ & n.a. & $3(60)$ & $\begin{array}{l}21 \\
(91.3)\end{array}$ & $0(0)$ \\
\hline $\begin{array}{l}\text { Education, } \\
\text { y }\end{array}$ & $\begin{array}{l}14.8 \\
(2.8)\end{array}$ & $\begin{array}{l}16.4 \\
(3.7)\end{array}$ & $\begin{array}{l}17.3 \\
(2.6)\end{array}$ & $\begin{array}{l}16.2 \\
(2.2)\end{array}$ & $\begin{array}{l}16.4 \\
(2.4)\end{array}$ & $\begin{array}{l}15.8 \\
(2.3)\end{array}$ & $\begin{array}{l}15.1 \\
(3.1)\end{array}$ & $\begin{array}{l}15.8 \\
(3.4)\end{array}$ & n.a. & $\begin{array}{l}15.2 \\
(1.8)\end{array}$ & $\begin{array}{l}15.4 \\
(3.1)\end{array}$ & $12(0)$ \\
\hline \multicolumn{13}{|c|}{ CSF measurements, mean (SD), pg/ml } \\
\hline PGRN & $\begin{array}{l}1515 \\
(295)\end{array}$ & $\begin{array}{l}1434 \\
(259)\end{array}$ & $\begin{array}{l}1546 \\
(323)\end{array}$ & $\begin{array}{l}1728 \\
(258)\end{array}$ & $\begin{array}{l}1648 \\
(433)\end{array}$ & $\begin{array}{l}1407 \\
(221)\end{array}$ & $\begin{array}{l}1535 \\
(442)\end{array}$ & $\begin{array}{l}1631 \\
(365)\end{array}$ & n.a. & $\begin{array}{l}1855 \\
(503)\end{array}$ & $\begin{array}{l}1671 \\
(441)\end{array}$ & $\begin{array}{l}2641 \\
(0)\end{array}$ \\
\hline$A \beta 42$ & $\begin{array}{l}1447 \\
(251)\end{array}$ & $\begin{array}{l}748 \\
(144)\end{array}$ & $\begin{array}{l}709 \\
(168)\end{array}$ & $\begin{array}{l}1760 \\
(676)\end{array}$ & $\begin{array}{l}1396 \\
(263)\end{array}$ & $\begin{array}{l}479 \\
(207)\end{array}$ & $\begin{array}{l}610 \\
(151)\end{array}$ & $\begin{array}{l}1637 \\
(464)\end{array}$ & n.a. & $\begin{array}{l}492 \\
(116)\end{array}$ & $\begin{array}{l}513 \\
(125)\end{array}$ & $\begin{array}{l}1147 \\
(0)\end{array}$ \\
\hline P-tau $181 p$ & $\begin{array}{l}17.1 \\
(2.6)\end{array}$ & $\begin{array}{l}16.0 \\
(3.2)\end{array}$ & $\begin{array}{l}33.9 \\
(9.7)\end{array}$ & $\begin{array}{l}26.5 \\
(3.9)\end{array}$ & $\begin{array}{l}16.2 \\
(2.7)\end{array}$ & $\begin{array}{l}16.6 \\
(4.2)\end{array}$ & $\begin{array}{l}37.9 \\
(12.0)\end{array}$ & $\begin{array}{l}29.8 \\
(12.0)\end{array}$ & n.a. & $\begin{array}{l}18.1 \\
(2.8)\end{array}$ & $\begin{array}{l}36.7 \\
(14.9)\end{array}$ & $70.5(0)$ \\
\hline T-tau & $\begin{array}{l}194 \\
(28)\end{array}$ & $\begin{array}{l}172 \\
(31)\end{array}$ & $\begin{array}{l}334 \\
(86)\end{array}$ & $\begin{array}{l}300 \\
(47)\end{array}$ & $\begin{array}{l}187 \\
(30)\end{array}$ & $\begin{array}{l}174 \\
(34)\end{array}$ & $\begin{array}{l}365 \\
(101)\end{array}$ & $\begin{array}{l}324 \\
(96)\end{array}$ & n.a. & $\begin{array}{l}200 \\
(26)\end{array}$ & $\begin{array}{l}362 \\
(110)\end{array}$ & $718(0)$ \\
\hline sTNFR1 & $\begin{array}{l}832 \\
(133)\end{array}$ & $\begin{array}{l}717 \\
(159)\end{array}$ & $\begin{array}{l}983 \\
(213)\end{array}$ & $\begin{array}{c}1071 \\
(187)\end{array}$ & $\begin{array}{l}798 \\
(174)\end{array}$ & $\begin{array}{l}597 \\
(130)\end{array}$ & $\begin{array}{l}871 \\
(191)\end{array}$ & $\begin{array}{l}1040 \\
(170)\end{array}$ & n.a. & $\begin{array}{l}649 \\
(63)\end{array}$ & $\begin{array}{l}877 \\
(174)\end{array}$ & $\begin{array}{l}1531 \\
(0)\end{array}$ \\
\hline sTNFR2 & $\begin{array}{l}967 \\
(170)\end{array}$ & $\begin{array}{l}844 \\
(177)\end{array}$ & $\begin{array}{l}1171 \\
(280)\end{array}$ & $\begin{array}{l}1199 \\
(199)\end{array}$ & $\begin{array}{l}921 \\
(193)\end{array}$ & $\begin{array}{l}749 \\
(103)\end{array}$ & $\begin{array}{l}1061 \\
(238)\end{array}$ & $\begin{array}{l}1227 \\
(272)\end{array}$ & n.a. & $\begin{array}{l}814 \\
(106)\end{array}$ & $\begin{array}{l}1061 \\
(240)\end{array}$ & $\begin{array}{l}1794 \\
(0)\end{array}$ \\
\hline TGF- $\beta 1$ & $\begin{array}{l}98.7 \\
(26.5)\end{array}$ & $\begin{array}{l}82.5 \\
(21.7)\end{array}$ & $\begin{array}{l}110 \\
(24.4)\end{array}$ & $\begin{array}{l}128 \\
(41.4)\end{array}$ & $\begin{array}{l}91.6 \\
(25.8)\end{array}$ & $\begin{array}{l}85.7 \\
(29.1)\end{array}$ & $\begin{array}{l}102 \\
(33.4)\end{array}$ & $\begin{array}{l}101 \\
(24.8)\end{array}$ & n.a. & $\begin{array}{l}76.8 \\
(29.8)\end{array}$ & $\begin{array}{l}106 \\
(28)\end{array}$ & $193(0)$ \\
\hline IL-10 & $5.7(2.8)$ & $4.9(1.4)$ & $5.1(1.6)$ & $6.3(2.1)$ & $5.3(1.7)$ & $5.9(3.1)$ & $5.6(2.5)$ & $5.8(3.0)$ & n.a. & $\begin{array}{l}5.1 \\
(2,5)\end{array}$ & $6.3(2.7)$ & $5.2(0)$ \\
\hline
\end{tabular}




\begin{tabular}{|c|c|c|c|c|c|c|c|c|c|c|c|c|}
\hline \multirow[t]{2}{*}{$N=965$} & \multicolumn{4}{|l|}{$\mathrm{CDR}=0$} & \multicolumn{4}{|l|}{$\mathrm{CDR}=0.5$} & \multicolumn{4}{|c|}{$C D R=1$} \\
\hline & $\begin{array}{l}\text { A-/TN- } \\
(n= \\
121)\end{array}$ & $\begin{array}{l}A+T N- \\
(n=52)\end{array}$ & $\begin{array}{l}A+/ T N+ \\
(n=45)\end{array}$ & $\begin{array}{l}A-/ T N+ \\
(n=73)\end{array}$ & $\begin{array}{l}\text { A-/TN- } \\
(n= \\
118)\end{array}$ & $\begin{array}{l}A+T N- \\
(n=89)\end{array}$ & $\begin{array}{l}\text { At/TN+ } \\
(n= \\
270)\end{array}$ & $\begin{array}{l}A-/ T N+ \\
(n=97)\end{array}$ & $\begin{array}{l}A-/ T N- \\
(n=2)\end{array}$ & $\begin{array}{l}A+T N- \\
(n= \\
12)\end{array}$ & $\begin{array}{l}A+/ T N+ \\
(n=78)\end{array}$ & $\begin{array}{l}\text { A-/TN+ } \\
(n=8)\end{array}$ \\
\hline ICAM1 & $\begin{array}{l}341 \\
(127)\end{array}$ & $\begin{array}{l}269 \\
(85)\end{array}$ & $\begin{array}{l}355 \\
(81)\end{array}$ & $\begin{array}{l}396 \\
(263)\end{array}$ & $\begin{array}{l}279 \\
(102)\end{array}$ & $\begin{array}{l}341 \\
(158)\end{array}$ & $\begin{array}{l}389 \\
(216)\end{array}$ & $\begin{array}{l}480 \\
(231)\end{array}$ & n.a. & $\begin{array}{l}397 \\
(189)\end{array}$ & $\begin{array}{l}357 \\
(177)\end{array}$ & $511(0)$ \\
\hline VCAM1 & $\begin{array}{l}35,016 \\
(10,943)\end{array}$ & $\begin{array}{l}29,069 \\
(11,269)\end{array}$ & $\begin{array}{l}55,843 \\
(33,183)\end{array}$ & $\begin{array}{l}61,573 \\
(22,084)\end{array}$ & $\begin{array}{l}39,565 \\
(13,438)\end{array}$ & $\begin{array}{l}31,629 \\
(11,705)\end{array}$ & $\begin{array}{l}38,723 \\
(14,869)\end{array}$ & $\begin{array}{l}56,287 \\
(24,005)\end{array}$ & n.a. & $\begin{array}{l}32,538 \\
(8,946)\end{array}$ & $\begin{array}{l}38,482 \\
(14,099)\end{array}$ & $\begin{array}{l}99,591 \\
(99,591)\end{array}$ \\
\hline IL-6 & $4.2(1.5)$ & $3.6(1.1)$ & $5.1(3.6)$ & $5.1(2.0)$ & $4.1(2.4)$ & $5.2(3.5)$ & $4.4(2.8)$ & $5.4(4.4)$ & n.a. & $\begin{array}{l}3.9 \\
(1.3)\end{array}$ & $4.5(3.1)$ & $3.3(0)$ \\
\hline IL-7 & $\begin{array}{l}1.03 \\
(0.74)\end{array}$ & $\begin{array}{l}1.05 \\
(0.67)\end{array}$ & $\begin{array}{l}0.95 \\
(0.65)\end{array}$ & $\begin{array}{l}1.10 \\
(1.12)\end{array}$ & $\begin{array}{l}1.05 \\
(1.01)\end{array}$ & $\begin{array}{l}1.33 \\
(0.72)\end{array}$ & $\begin{array}{l}1.46 \\
(1.25)\end{array}$ & $\begin{array}{l}0.91 \\
(0.55)\end{array}$ & & $\begin{array}{l}0.75 \\
(0.40)\end{array}$ & $\begin{array}{l}1.38 \\
(0.97)\end{array}$ & $1.50(0)$ \\
\hline
\end{tabular}

Abbreviations: PGRN: progranulin; sTNFR: soluble tumor necrosis factor receptor; ICAM1: intercellular cell adhesion molecule-1; VCAM1: vascular cell adhesion molecule-1; IL: interleukin; CSF: cerebrospinal fluid; A $\beta$ : $\beta$-amyloid; CDR: clinical dementia rating

In total samples, CSF PGRN was significantly higher in TN + profile $(p<0.0001$, Table e-2). The association remained significant irrespective of the presence of amyloid pathology, within either $C D R=0(F=29, p<0.0001$ for $A$ - group and $F=7.95, p=0.004$ for $A+$ group $)$ or $C D R=0.5(F=8.47, p=0.002$ for $A$ - group and $F=8.67, p=0.004$ for $A+$ group) group. The $C D R=1$ group did not yield a sufficient number of subjects per $A / T / N$ profile to allow for group comparisons. (Fig. 1) In contrast, CSF PGRN was significantly lower in $A+$ profile $(p<0.0001)$. The difference remained significant only in TN + profile $(F=9.59, p=0.002$ for $C D R=0$ group and $F=11, p=0.0001$ for $C D R=0.5$ group). Within the $C D R=1.0$ group, the abovementioned difference showed a trend toward significance in $\mathrm{TN}+$ profile $(\mathrm{p}=0.07)$.

\subsection{Specific neuroinflammatory markers were elevated following neuronal damages}

We next tested the distribution pattern of CSF neuroinflammatory marker within the framework in 228 ADNI participants (Table 1). Different groups according to the combination of A/TN classification and clinical stage are summarized in Table e-1.

The levels of five neuroinflammatory markers (sTNFR1, sTNFR2, TGF- 31 , VCAM1, and ICAM1) were significantly elevated in TN-positive individuals (Table e-2). Specifically, within the CDR = 0 group, levels of sTNFR1, sTNFR2, and VCAM1 were significantly increased in TN + profile. Similar trends were identified in the CDR $=0.5$ group, such that levels of sTNFR1, sTNFR2, TGF- $\beta 1$, VCAM1, and ICAM1 were significantly elevated in TN + profile. Notably, the associations with several markers (including sTNFR1, sTNFR2, and VCAM1) were significant, independent of the A status, while the relationships of TGF$\beta 1$ and ICAM1 were significant only in A+/TN+. Within the CDR = 1 group, levels of TNFR1, TNFR2, TGF- $\beta 1$, and VCAM1 were significantly higher in A+/TN+, compared with those in A+/TN- profile. (Fig. 1) No significant differences were identified for IL-6 and IL-7 (Table e-3).

\subsection{PGRN was associated with increased neuroinflammatory markers following neuronal damages}

Since both PGRN and specific neuroinflammatory markers were elevated following the tau-related neurodegeneration, we further examined whether PGRN could be a neuroinflammatory marker following neuronal damages. PGRN was positively related to the abovementioned markers (including sTNFR1, sTNFR2, TGF- $\beta 1$, ICAM1, and VCAM1) and the associations were significant only in TN + profile (Fig. 2A to 2E). Interestingly, PGRN showed significant associations with ICAM1 and TGF- $\beta 1$ only in A+/TN + profile. These findings highlighted that neuronal damages might be an upstream trigger which promoted PGRN and specific neuroinflammation.

\subsection{PGRN protected against amyloid burden via mediating tau-related neuroinflammation.}

We further asked how these responses (elevation of PGRN as well as neuroinflammatory molecules) could influence amyloid pathology. We found the association of CSF PGRN (Fig. 3A) and CSF neuroinflammatory markers (Fig. 3B to 3F) with CSF Aß42 were only significant (or stronger) in TN + group. Moreover, PGRN could alleviate cerebral amyloid deposition via promoting these neuroinflammatory markers, including sTNFR1 $(p r o p o r t i o n=50.3 \%, p=$ 0.006), sTNFR2 (proportion $=28.4 \%, p=0.01$ ), and VCAM1 (proportion $=44.2 \%, p=0.008$ ) (Figure e-1A). Within the A-T-N framework, the abovementioned mediation effects of neuroinflammation (sTNFR1, sTNFR2, and VCAM1) were significant only in TN + profile, with the mediation proportion ranged from 30-60\% (Fig. 3G). Interestingly, we also found significant but smaller (10\% 30\%) mediation effects of CSF PGRN in influencing association of CSF neuroinflammatory markers (sTNFR1, sTNFR2, and ICAM1) with CSF AB42 in TN + profile (Fig. 3H). Similar results were obtained in A+/TN + group (Fig. 3I): ICAM1 and TGF- $\beta 1$ were specifically revealed as mediating molecules for the association between PGRN and amyloid burden in A+/TN + group (Figure e-2.

\subsection{PGRN protected against cognitive impairments.}


Finally, we explored whether CSF levels of PGRN could predict longitudinal changes of cognitive functions. We found protective roles of CSF PGRN in cognitive function, including the general cognition $(p=0.008$, Fig. $4 A)$, memory function $(p=0.0002$, Fig. 4B), and executive function ( $p=0.028$, Fig. 4C) in TN + profile, but not in TN-profile. These indicated that PGRN might serve as a responding molecule that actively fight against the neuronal injuries.

\section{Discussion}

Herein, we explored the relationships of PGRN with neuroinflammatory makers and evaluated the roles of neuroinflammation in mediating the influences of PGRN on amyloid pathology and cognition. Finally, we found PGRN could protect against amyloid pathology and cognitive impairments via mediating specific neuroinflammatory markers. These findings supported the guardian roles of PGRN, a marker of lysosomal functioning [1], in fighting against AD via modulating neuroinflammation occurring with neurodegeneration.

PGRN was proposed to be a hallmark of microglia-mediated neuroinflammation [10]. Similar with PGRN, CSF sTREM2, a marker of microglial activation, was found to be elevated in early AD with TN + profile [16]. It was also reported that CSF PGRN was associated with CSF sTREM2 only in AD and SNAP groups [10], suggesting PGRN might be a hallmark of neuroinflammation occurring with neurodegeneration. Though no causal conclusion can be made due to the cross-sectional design, these findings indicated a close relationship between tau load and enhanced neuroinflammation. Future studies were warranted to explore the differences in the inflammatory processes induced by amyloid and tau, and their influences on development of neurodegeneration.

Neuroinflammation plays a critical role in modulating AD pathologies. We and other teams previously reported increased peripheral levels of sTNFR1, sTNFR2 $[9,26,27]$ and IL- 6 , as well as elevated CSF levels of IL-10 and TGF- $\beta 1$ in AD compared with the controls [9]. TNFRs could be activated by binding of soluble TNF, a hallmark of neuroinflammation as well as neurodegenerative conditions [28], and could be cleaved to generate sTNFRs. The circulating levels of sTNFR were positively associated with the levels of plasma amyloid and tau [26, 27] and the conversion rate to dementia [27]. Another study on transgenic mice showed TNFR1 deletion reduced Aß pathology, microglia activation, neuron loss, and memory deficits [29]. In concordance with the present study, previous studies found CSF levels of ICAM1 and VCAM1 were increased during the preclinical and prodromal stages of $A D[30,31]$.

It was found that PGRN could suppress neuroinflammation following induced toxic stimuli or injury [5, 32]. The present study revealed PGRN might be a hallmark of tau-related neuroinflammatory activities (including anti- and pro-inflammatory markers), which were favorable for alleviating amyloid pathology. This suggested that neuroinflammation in CNS might play a "double-edged sword" role in dealing with neurodegeneration. Similar clues were reported for microglia, which adopted numerous fates with homeostatic microglia and a microglial neurodegenerative phenotype representing two opposite ends [33]. In addition, the impacts of PGRN on cerebral amyloid metabolism were conflicting [4, 12], possibly because the TN status were not considered.

\section{Limitation}

Some caution is warranted given that several limitations existed for the present study. First, the sample size is not large enough in some groups and we thus conducted analyses in total sample after adjusting for A/TN profile or CDR. Second, causal mediation associations only reflect but cannot equal to the causal relationships. Longitudinal analyses should be conducted to confirm our findings about the influences of PGRN on neuroinflammatory markers and $A D$ pathologies in well-characterized samples. Third, in vivo and in vitro studies are warranted to examine the influences of neuronal injuries on or tau-related neurodegeneration on PGRN, neuroinflammatory markers, and amyloid metabolism.

\section{Conclusions}

In conclusion, we demonstrated PGRN was not only a marker of neuroinflammatory activities occurring with neuronal injuries, but could alleviate the cerebral amyloid- $\beta$ burden and cognitive impairments via neuroinflammatory mechanisms. These findings warrant further validation in larger, wellcharacterized samples.

\section{Abbreviations}

PGRN

progranulin

CNS

central nervous system

$A D$

Alzheimer's disease

STNFR

soluble tumor necrosis factor receptor

ICAM1

intercellular cell adhesion molecule-1

VCAM1 
vascular cell adhesion molecule-1

IL

interleukin

CSF

cerebrospinal fluid

$A \beta$

$\beta$-amyloid

ADNI

Alzheimer's Disease Neuroimaging Initiative

CDR

clinical dementia rating

ELISA

enzyme-linked immunosorbent assay

MSD

mass spectrometry detector

CV

coefficient of variation

ECLIA

electrochemiluminescence immunoassays

ADAS

Alzheimer's disease assessment scale

RAVLT

Rey auditory verbal learning test

MMSE

Mini-Mental State Examination

ANCOVAs

one-way analyses of covariance

\section{Declarations}

\section{Ethics approval and consent to participate}

ADNI was approved by institutional review boards of all participating institutions, and written informed consent was obtained from all participants or their guardians.

\section{Consent for publication}

Not applicable

Availability of data and materials

The datasets used and/or analyzed during the current study are available from http://adni.loni.usc.edu/

\section{Competing interests}

The authors declare that they have no competing interests.

\section{Funding}

Not applicable

\section{Authors' contributions}

Dr. Wei Xu: conceptualization and design of the study, collection and analysis of the data, drafting and revision of the manuscript, and prepared all the figures. Dr. Chen-Chen Tan, MS. Xi-Peng Cao, Prof. Jin-Tai Yu and Prof. Lan Tan: revision of the manuscript.

\section{Acknowledgements}


We want thank for all the contributions of the participants from the Alzheimer's Disease Neuroimaging Initiative (ADNI) (National Institutes of Health Grant U01 AG024904) and DOD ADNI (Department of Defense award number W81XWH-12-2-0012). ADNI is funded by the National Institute on Aging, the National Institute of Biomedical Imaging and Bioengineering, and through generous contributions from the following: AbbVie, Alzheimer's Association; Alzheimer's Drug Discovery Foundation; Araclon Biotech; BioClinica, Inc.; Biogen; Bristol-Myers Squibb Company; CereSpir, Inc.; Cogstate; Eisai Inc.; Elan Pharmaceuticals, Inc.; Eli Lilly and Company; Eurolmmun; F. Hoffmann-La Roche Ltd and its affiliated company Genentech, Inc.; Fujirebio; GE Healthcare; IXICO Ltd.; Janssen Alzheimer Immunotherapy Research \& Development, LLC.; Johnson \& Johnson Pharmaceutical Research \& Development LLC.; Lumosity; Lundbeck; Merck \& Co., Inc.; Meso Scale Diagnostics, LLC.; NeuroRx Research; Neurotrack Technologies; Novartis Pharmaceuticals Corporation; Pfizer Inc.; Piramal Imaging; Servier; Takeda Pharmaceutical Company; and Transition Therapeutics. The Canadian Institutes of Health Research is providing funds to support ADNI clinical sites in Canada. Private sector contributions are facilitated by the Foundation for the National Institutes of Health (www.fnih.org). The grantee organization is the Northern California Institute for Research and Education, and the study is coordinated by the Alzheimer's Therapeutic Research Institute at the University of Southern California. ADNI data are disseminated by the Laboratory for Neuro Imaging at the University of Southern California.

\section{References}

1. Paushter DH, Du H, Feng T, Hu F. The lysosomal function of progranulin, a guardian against neurodegeneration. Acta Neuropathol. 2018;136:1-17.

2. Gotzl JK, Colombo AV, Fellerer K, Reifschneider A, Werner G, Tahirovic S, Haass C, Capell A. Early lysosomal maturation deficits in microglia triggers enhanced lysosomal activity in other brain cells of progranulin knockout mice. Mol Neurodegener. 2018;13:48.

3. Chang MC, Srinivasan K, Friedman BA, Suto E, Modrusan Z, Lee WP, Kaminker JS, Hansen DV, Sheng M. Progranulin deficiency causes impairment of autophagy and TDP-43 accumulation. J Exp Med. 2017;214:2611-28.

4. Takahashi H, Klein ZA, Bhagat SM, Kaufman AC, Kostylev MA, Ikezu T, Strittmatter SM. Alzheimer's Disease Neuroimaging I: Opposing effects of progranulin deficiency on amyloid and tau pathologies via microglial TYROBP network. Acta Neuropathol. 2017;133:785-807.

5. Ma Y, Matsuwaki T, Yamanouchi K, Nishihara M. Progranulin Protects Hippocampal Neurogenesis via Suppression of Neuroinflammatory Responses Under Acute Immune Stress. Mol Neurobiol. 2017;54:3717-28.

6. Wu Z, Ji S, Zheng J, Hu Z, Xiao S, Wei Y, Zhuo Z, Lin Y, Yang W, Xu K, et al. Prelithiation Activates Li(Ni0.5Mn0.3Co0.2)O2 for High Capacity and Excellent Cycling Stability. Nano Lett. 2015;15:5590-6.

7. Xu HM, Tan L, Wan Y, Tan MS, Zhang W, Zheng ZJ, Kong LL, Wang ZX, Jiang T, Tan L, Yu JT. PGRN Is Associated with Late-Onset Alzheimer's Disease: a Case-Control Replication Study and Meta-analysis. Mol Neurobiol. 2017;54:1187-95.

8. Calsolaro V, Edison P. Neuroinflammation in Alzheimer's disease: Current evidence and future directions. Alzheimers Dement. 2016;12:719-32.

9. Shen XN, Niu LD, Wang YJ, Cao XP, Liu Q, Tan L, Zhang C, Yu JT. Inflammatory markers in Alzheimer's disease and mild cognitive impairment: a meta-analysis and systematic review of 170 studies. J Neurol Neurosurg Psychiatry. 2019;90:590-8.

10. Suarez-Calvet M, Capell A, Araque Caballero MA, Morenas-Rodriguez E, Fellerer K, Franzmeier N, Kleinberger G, Eren E, Deming Y, Piccio L, et al: CSF progranulin increases in the course of Alzheimer's disease and is associated with sTREM2, neurodegeneration and cognitive decline. EMBO Mol Med $2018,10$.

11. Mendsaikhan A, Tooyama I, Bellier JP, Serrano GE, Sue LI, Lue LF, Beach TG, Walker DG. Characterization of lysosomal proteins Progranulin and Prosaposin and their interactions in Alzheimer's disease and aged brains: increased levels correlate with neuropathology. Acta Neuropathol Commun. 2019;7:215.

12. Minami SS, Min SW, Krabbe G, Wang C, Zhou Y, Asgarov R, Li Y, Martens LH, Elia LP, Ward ME, et al. Progranulin protects against amyloid beta deposition and toxicity in Alzheimer's disease mouse models. Nat Med. 2014;20:1157-64.

13. Petersen RC, Aisen PS, Beckett LA, Donohue MC, Gamst AC, Harvey DJ, Jack CR Jr, Jagust WJ, Shaw LM, Toga AW, et al. Alzheimer's Disease Neuroimaging Initiative (ADNI): clinical characterization. Neurology. 2010;74:201-9.

14. Trojanowski JQ, Vandeerstichele H, Korecka M, Clark CM, Aisen PS, Petersen RC, Blennow K, Soares H, Simon A, Lewczuk P, et al. Update on the biomarker core of the Alzheimer's Disease Neuroimaging Initiative subjects. Alzheimers Dement. 2010;6:230-8.

15. Weiner MW, Aisen PS, Jack CR Jr, Jagust WJ, Trojanowski JQ, Shaw L, Saykin AJ, Morris JC, Cairns N, Beckett LA, et al. The Alzheimer's disease neuroimaging initiative: progress report and future plans. Alzheimers Dement. 2010;6:202-11 e207.

16. Suarez-Calvet M, Morenas-Rodriguez E, Kleinberger G, Schlepckow K, Araque Caballero MA, Franzmeier N, Capell A, Fellerer K, Nuscher B, Eren E, et al. Early increase of CSF sTREM2 in Alzheimer's disease is associated with tau related-neurodegeneration but not with amyloid-beta pathology. Mol Neurodegener. 2019;14:1.

17. Jack CR Jr, Bennett DA, Blennow K, Carrillo MC, Dunn B, Haeberlein SB, Holtzman DM, Jagust W, Jessen F, Karlawish J, et al. NIA-AA Research Framework: Toward a biological definition of Alzheimer's disease. Alzheimers Dement. 2018;14:535-62.

18. Jack CR Jr, Bennett DA, Blennow K, Carrillo MC, Feldman HH, Frisoni GB, Hampel H, Jagust WJ, Johnson KA, Knopman DS, et al. A/T/N: An unbiased descriptive classification scheme for Alzheimer disease biomarkers. Neurology. 2016;87:539-47.

19. Hansson O, Seibyl J, Stomrud E, Zetterberg H, Trojanowski JQ, Bittner T, Lifke V, Corradini V, Eichenlaub U, Batrla R, et al. CSF biomarkers of Alzheimer's disease concord with amyloid-beta PET and predict clinical progression: A study of fully automated immunoassays in BioFINDER and ADNI cohorts. Alzheimers Dement. 2018;14:1470-81. 
20. Shaw LM, Vanderstichele H, Knapik-Czajka M, Clark CM, Aisen PS, Petersen RC, Blennow K, Soares H, Simon A, Lewczuk P, et al. Cerebrospinal fluid biomarker signature in Alzheimer's disease neuroimaging initiative subjects. Ann Neurol. 2009;65:403-13.

21. Capell A, Liebscher S, Fellerer K, Brouwers N, Willem M, Lammich S, Gijselinck I, Bittner T, Carlson AM, Sasse F, et al. Rescue of progranulin deficiency associated with frontotemporal lobar degeneration by alkalizing reagents and inhibition of vacuolar ATPase. J Neurosci. 2011;31:1885-94.

22. Gibbons LE, Carle AC, Mackin RS, Harvey D, Mukherjee S, Insel P, Curtis SM, Mungas D, Crane PK. Alzheimer's Disease Neuroimaging I: A composite score for executive functioning, validated in Alzheimer's Disease Neuroimaging Initiative (ADNI) participants with baseline mild cognitive impairment. Brain Imaging Behav. 2012;6:517-27.

23. Crane PK, Carle A, Gibbons LE, Insel P, Mackin RS, Gross A, Jones RN, Mukherjee S, Curtis SM, Harvey D, et al. Development and assessment of a composite score for memory in the Alzheimer's Disease Neuroimaging Initiative (ADNI). Brain Imaging Behav. 2012;6:502-16.

24. Baron RM, Kenny DA. The moderator-mediator variable distinction in social psychological research: conceptual, strategic, and statistical considerations. J Pers Soc Psychol. 1986;51:1173-82.

25. Imai K, Keele L, Tingley D. A general approach to causal mediation analysis. Psychol Methods. 2010;15:309-34.

26. Zhang J, Peng M, Jia J. Plasma amyloid-beta oligomers and soluble tumor necrosis factor receptors as potential biomarkers of AD. Curr Alzheimer Res. 2014;11:325-31.

27. Buchhave P, Zetterberg H, Blennow K, Minthon L, Janciauskiene S, Hansson O. Soluble TNF receptors are associated with Abeta metabolism and conversion to dementia in subjects with mild cognitive impairment. Neurobiol Aging. 2010;31:1877-84.

28. McCoy MK, Tansey MG. TNF signaling inhibition in the CNS: implications for normal brain function and neurodegenerative disease. J Neuroinflammation. 2008;5:45.

29. He P, Zhong Z, Lindholm K, Berning L, Lee W, Lemere C, Staufenbiel M, Li R, Shen Y. Deletion of tumor necrosis factor death receptor inhibits amyloid beta generation and prevents learning and memory deficits in Alzheimer's mice. J Cell Biol. 2007;178:829-41.

30. Janelidze S, Mattsson N, Stomrud E, Lindberg O, Palmqvist S, Zetterberg H, Blennow K, Hansson O. CSF biomarkers of neuroinflammation and cerebrovascular dysfunction in early Alzheimer disease. Neurology. 2018;91:e867-77.

31. Rauchmann BS, Sadlon A, Perneczky R, Alzheimer's Disease Neuroimaging I. Soluble TREM2 and Inflammatory Proteins in Alzheimer's Disease Cerebrospinal Fluid. J Alzheimers Dis. 2020;73:1615-26.

32. Martens LH, Zhang J, Barmada SJ, Zhou P, Kamiya S, Sun B, Min SW, Gan L, Finkbeiner S, Huang EJ, Farese RV Jr. Progranulin deficiency promotes neuroinflammation and neuron loss following toxin-induced injury. J Clin Invest. 2012;122:3955-9.

33. Gotzl JK, Brendel M, Werner G, Parhizkar S, Sebastian Monasor L, Kleinberger G, Colombo AV, Deussing M, Wagner M, Winkelmann J, et al: Opposite microglial activation stages upon loss of PGRN or TREM2 result in reduced cerebral glucose metabolism. EMBO Mol Med $2019,11$.

\section{Figures}




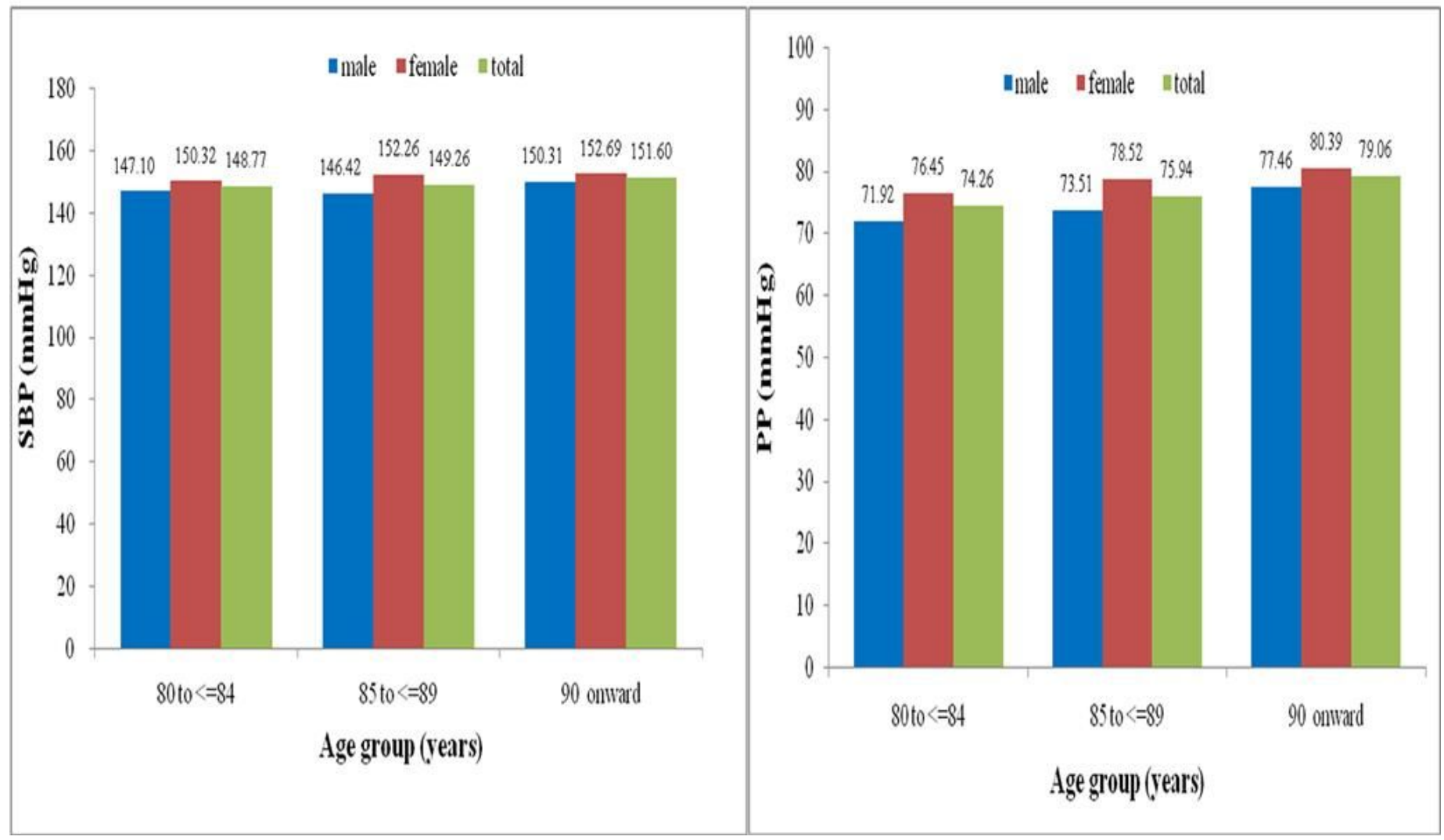

\section{Figure 1}

Early increases of CSF five neuroinflammatory markers and PGRN were associated with tau-related neurodegeneration but not amyloid pathology Scatter plot depicting CSF levels of five neuroinflammatory markers (including sTNFR1, sTNFR2, TGF- $\beta 1$, ICAM1, and VCAM1) as well as PGRN for each of the four biomarker profiles, as defined by the A/T/N framework, coupled with clinical staging, as defined by CDR. The T (tau pathology) and $\mathrm{N}$ (neurodegeneration) group were merged because these two biomarker groups were highly correlated. The CDR = 1 stage group includes some biomarker profiles containing insufficient number of subjects, which precludes conducting group comparison. They are still shown for sake of completeness. Each $\mathrm{A} / \mathrm{T} / \mathrm{N}$ biomarker profile is represented in a different color: A-/TN- are depicted in purple, A+/TN- in blue, A+/TN+ in grey, and A-/TN+ in orange. Solid bars represent the mean and the standard deviation (SD). P-values were assessed by one-way ANCOVAs adjusted for age, gender, and educational level. Pvalues were reported in bold after surviving Bonferroni correction (four comparisons for each CDR group). Abbreviations: A: $A \beta$ pathology biomarker status; T: tau pathology biomarker status AD; N: neurodegeneration biomarker status; Alzheimer's disease; CDR: clinical dementia rating; CSF: cerebrospinal fluid. 
Figure 2

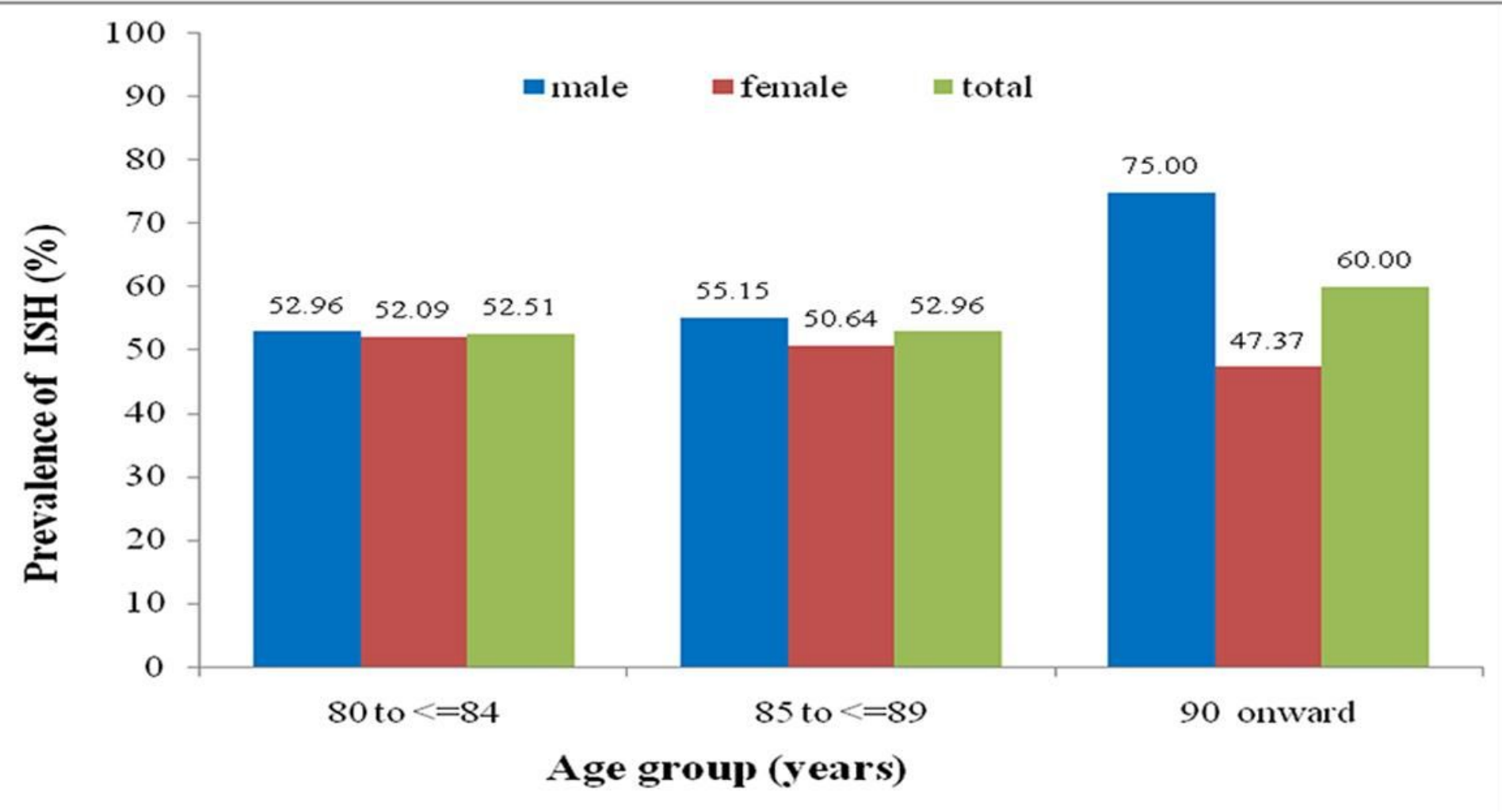

\section{Figure 2}

Associations of CSF PGRN with CSF neuroinflammatory markers The $x$ axis represents CSF PGRN level and the $y$ axis represents CSF specific neuroinflammatory marker level. Each $\mathrm{A} / \mathrm{T} / \mathrm{N}$ biomarker profile is represented in a different color: $\mathrm{A}-/ \mathrm{TN}$ - are depicted in green, $\mathrm{A}+/ \mathrm{TN}$ - in blue, $\mathrm{A}+/ \mathrm{TN}+$ in dark red, and $\mathrm{A}-/ \mathrm{TN}+$ in orange. P-values were assessed by multiple linear regression models adjusted for age, gender, education, and $\mathrm{CDR}$ stage. 


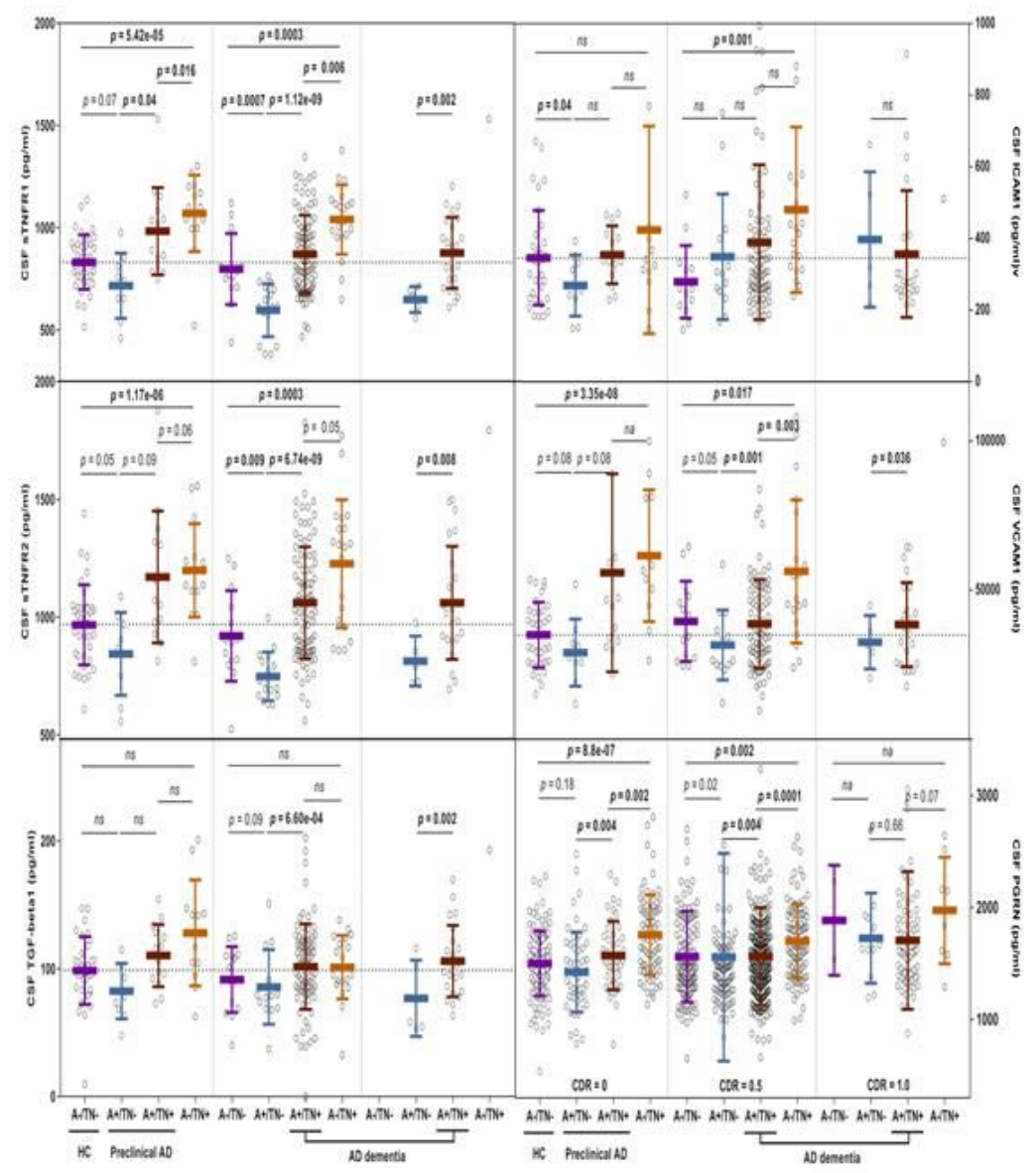

Figure 3

PGRN could buffer cerebral amyloid- $\beta$ burden via mediating tau-related neuroinflammation The association of CSF PGRN (3A) and abovementioned CSF neuroinflammatory markers (3B to $3 \mathrm{~F}$ ) with CSF A 342 were only significant or stronger in $\mathrm{TN}+$ group compared to TN- group. The mediation effects of neuroinflammatory markers (including sTNFR1, sTNFR2, and VCAM1) on the relationships of CSF PGRN with CSF AB42 were significant only in TN+ profile, with the mediation proportion ranged from $30 \%$ to $60 \%(3 \mathrm{G})$. Interestingly, we also found smaller (10\% 30\%) but significant mediation effects of CSF PGRN in influencing association of CSF neuroinflammatory markers (sTNFR1, sTNFR2, and ICAM1) with CSF AB42 in TN+ profile (3H). Similar results were obtained in A+/TN+ group (3I). P-values were adjusted for age, gender, education, APOE4 status, and CDR stage.

A

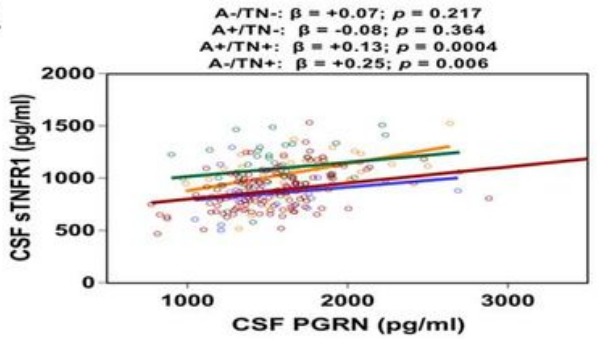

D

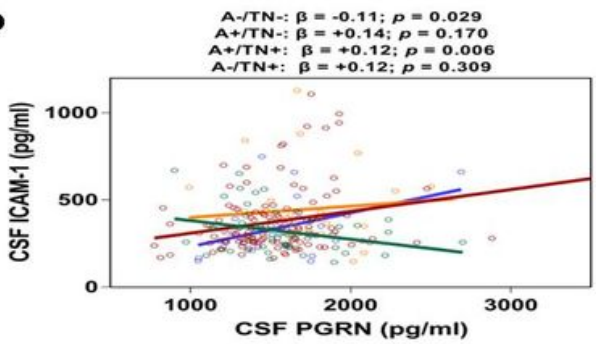

B

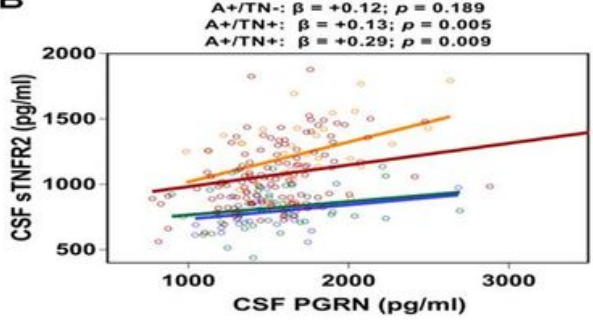

E

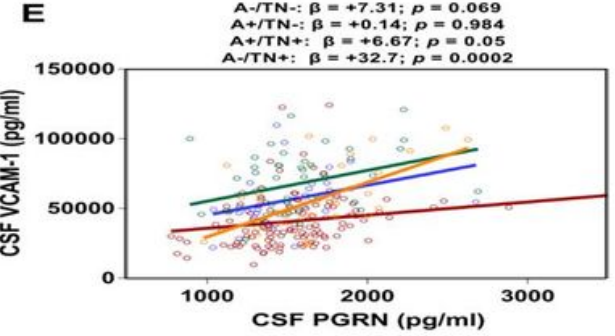

C

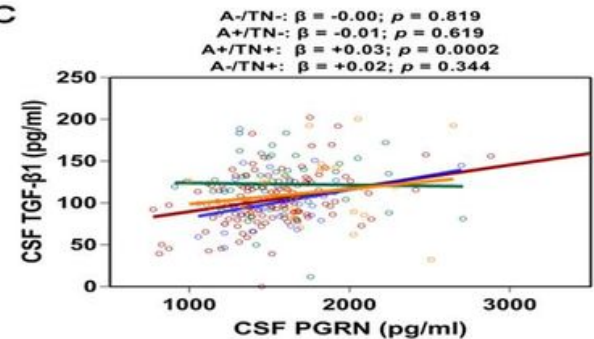

$=\stackrel{A-r T N-}{A+/ T N}$

$A+r T N+$

Figure 4

Longitudinal influences of CSF PGRN on the cognitive functions, stratified by the TN profile CSF PGRN were categorized into three tertiles (low, moderate, and high level) in order to facilitate the drawing. P-values were assessed by mixed-effect models adjusted for age, gender, education, APOE4 status, CDR, 
and amyloid status (A profile). We found protective roles of high CSF levels of PGRN in preventing decline of cognitive functions, including the general cognition (A), memory function (B), and executive function (C), in TN+ but no TN-profile.

\section{Supplementary Files}

This is a list of supplementary files associated with this preprint. Click to download.

- Supplementaryfile.pdf 